\title{
APPLICATION OF KNITTING STRUCTURE TEXTILES IN MEDICAL AREAS
}

\author{
Xiaohui Zhang ${ }^{1,3}$, Pibo Ma $\mathrm{Ma}^{1,2,3 *}$ \\ ${ }^{1}$ Engineering Research Center for Knitting Technology, Ministry of Education, Jiangnan University, Wuxi 214122, China \\ ${ }^{2}$ International Joint Research Laboratory for Novel Knitting Structural Materials, Jiangnan University, Wuxi 214122, China \\ ${ }^{3}$ Key Laboratory of Eco-textiles, Ministry of Education, Ministry of Education, Jiangnan University, Wuxi 214122, China \\ *Corresponding author: mapibo@jiangnan.edu.cn
}

\begin{abstract}
:
There are many kinds of medical textiles, such as woven textiles, non-woven textiles, braided textiles and knitted textiles. Non-woven medical textiles constitute more than $60 \%$ of the total medical textiles used, but are almost disposable ordinary medical textiles. While knitted fabrics forms a small part of the medical textiles, but are greatly applied in high-tech medical textiles, containing artificial blood vessels, hernia patches, cardiac support devices, knitted medical expandable metallic stents and tendon scaffolds. Knitting structures, including weft knitting structure and warp knitting structure. The knitted textiles are popular for their loose structure, greater flexibility, higher porosity, more flexible structure and better forming technology. The present article will introduce some knitting structures and materials applied in the medical textiles in accordance with non-implantable, implantable, extra-corporeal textiles and healthcare and hygiene products.
\end{abstract}

\section{Keywords:}

medical textiles, knitting structure, warp-knitted fabric, weft-knitted fabric

\section{Introduction}

Medical textiles are textiles that are used in mass care and patient treatment in medical institutions. It is a new field of combination of textile technology and medical science. Medical textiles are textiles with high-added value textile structures and products [1]. Textile materials have great practical value in medical field. The earliest medical textile materials are natural fibers, such as cotton and ramie. And with the advancement in technology, synthetic fiber is taking on a higher percentage in medical textiles. Now, biodegradable fibers are being developed and applied in medical materials.

According to their use, medical textiles are divided into nonimplantable, implantable, extra-corporeal textiles and healthcare and hygiene products. And according to their structure, medical textiles are classified into knitted fabric, woven fabric, braided fabric and non-woven fabric. Non-woven medical textiles constitute more than $60 \%$ of the total medical textiles used. They are almost disposable ordinary medical textiles. Knitted fabric, woven fabric and braided fabric make up a lower percentage of total medical fabric. However, they are the basic structures to create high-tech medical textiles, such as artificial blood vessels, medical patches and scaffolds for tissue engineering.

As compared with woven fabrics, knitted fabrics have loose structure, good flexibility, high porosity, flexible and changeable structure design. And the structures of knitted fabric can be varied to meet different requirements. They are very suitable for medical textiles, especially high-tech medical textiles. Weftknitted fabric applied in medical field includes medical dressings, bandages, padding of medical mattress, clothes for surgery and some high-tech products. Warp-knitted fabric is greatly used as mattress, covering materials and medical organ.

\section{Knitting structure}

Knitting is done by a set of connected loops from a series of yarn in warp or weft direction. Therefore, there are two main types of knitted fabric: weft knitted fabric and warp knitted fabric. In a weft knitted fabric, a horizontal row of loops are made using one thread that runs in the horizontal direction. While in a warp knitted fabric, each loop in the horizontal direction is made from different threads. Different types of machines, structures, stitch types, yarns and needle sizes may be used to create knitted fabrics with different properties and applications.

Knitting is known for its forming technology and the greater performances to knit mesh and spacer fabric. The features of forming technology are higher production efficiency, various patterns and flexible structures. It just needs one step to realize the process from fibers or yarns to fabrics. And the materials and structures have many choices to meet different requirements in application.

Knitting meshes include weft-knitted mesh and warp-knitted mesh. Table 1 shows some structures of knitted meshes. Fabrics with meshes often have great breathability, permeability and moisture conductivity. And the sizes of meshes are flexible and depend on the requirements. Spacer fabric has a threedimensional structure and can be knitted on both weft knitting machine and warp knitting machine. Spacer fabric often has certain thickness. The thickness is changeable and relies on the applications, which gives the fabric greater breathability and elastic resilience. Figure 3 is a weft-knitted spacer fabric and Figure 4 is a warp-knitted spacer fabric. 


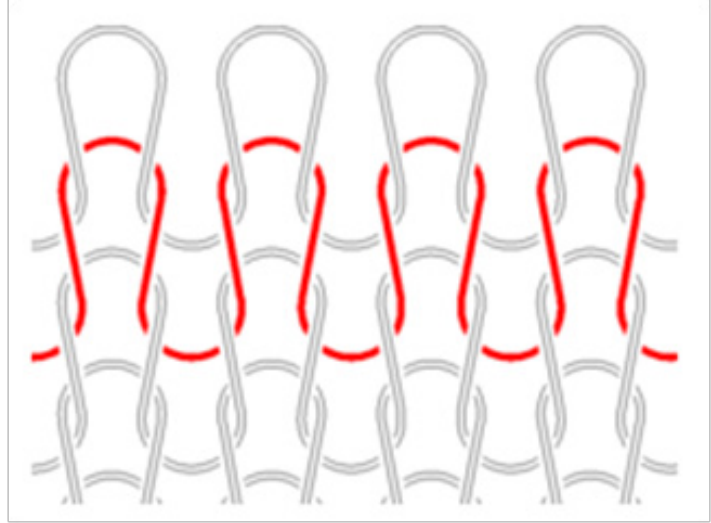

Figure 1. Basic pattern of weft knitting

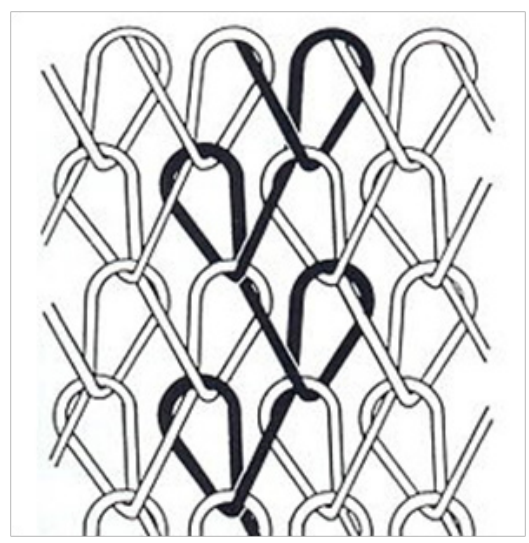

Figure 2. Basic pattern of warp knitting

Table 1. Some structures of the knitted meshes

\begin{tabular}{|c|c|c|c|c|}
\hline \multicolumn{2}{|c|}{ Weft-knitted meshes } & \multicolumn{3}{c|}{ Warp-knitted meshes } \\
\hline Tuck stitch & Dummy stitch & Elliptic mesh & Diamond mesh & Hexagonal mesh \\
\hline & & & & \\
\hline
\end{tabular}

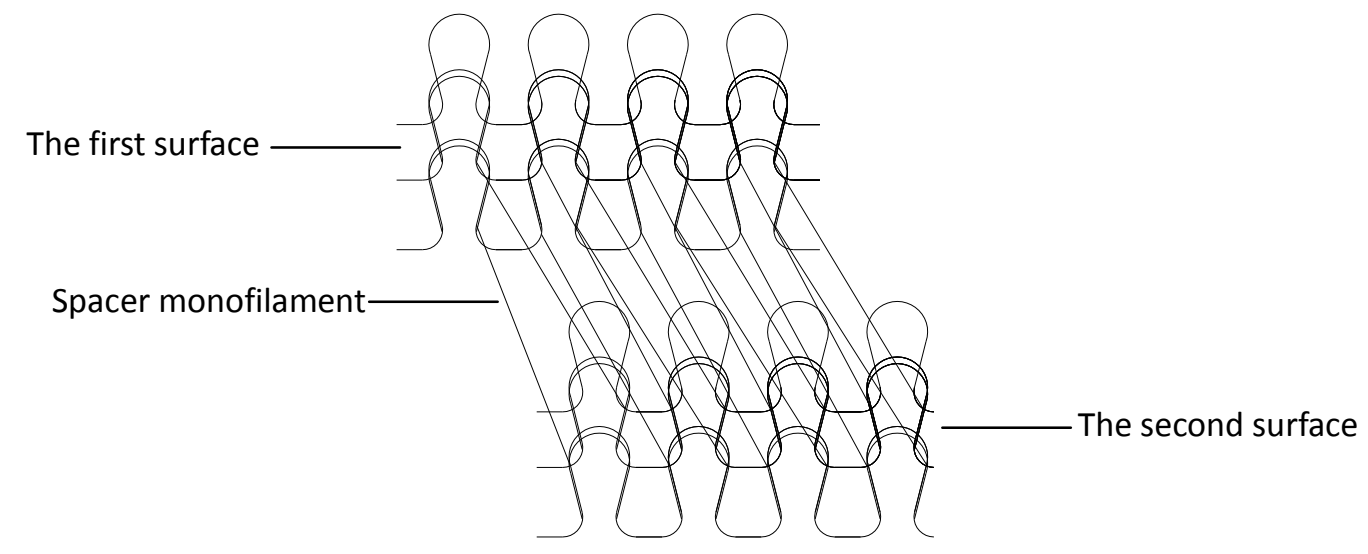

Figure 3. Weft-knitted spacer fabric

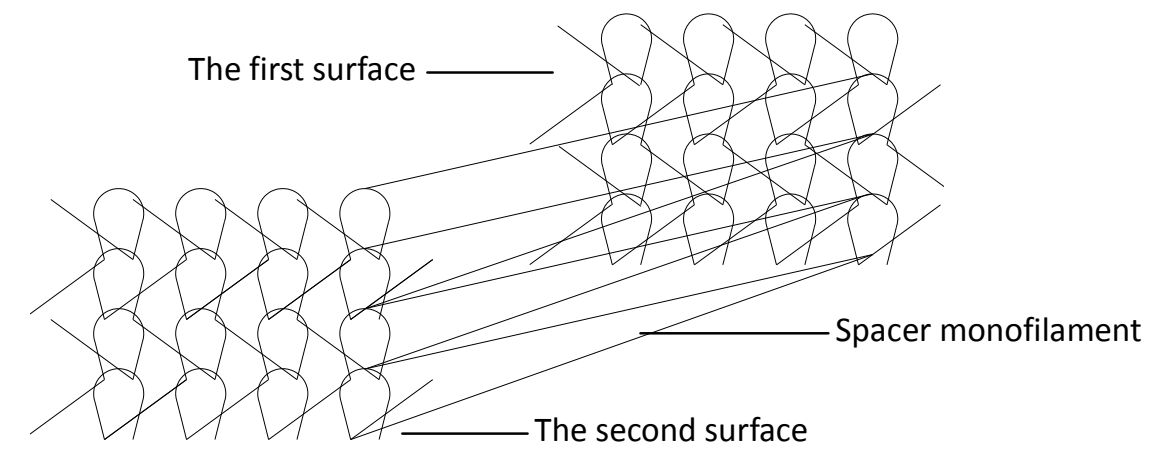

Figure 4.Warp-knitted spacer fabric 


\section{Knitted medical textiles}

\subsection{Non-implantable textiles}

Non-implantable textiles mainly include medical dressings, gauze, bandages and other textiles that come in contact with the skin wound. They are used to cover and protect wounds, prevent infection and promote the role of healing.

\subsubsection{Medical dressings}

The action of medical dressings is to insulate and prevent trauma, attach the drugs to the wound and absorb liquids. It is essential for wound dressings to have the properties of good hygroscopicity, good breathability and great sense of comfort when it comes in contact with the skin. The knitted medical dressings show greater extensibility, elasticity, fitness and flexibility.

For two dimensional structures, weft plain stitch and rib stitch are greatly applied in medical dressings for simple knitting technology, great flexibility and low viscidity. Besides these, some three dimensional structures, including weft multiply composites, weft knitted spacer fabrics and warp knitted spacer fabrics, are greatly used in the field of medical dressings. They often have absorbent layers for good ability to control heat and moisture transfer. And the materials applied in medical dressings refer to cotton, viscose filament, alginate fiber, jutecell and chitosan. Yarns and filaments take great portion for little loss of short fiber during the procedure.

\subsubsection{Weft plain stitch applied in medical dressings}

Medical dressings based on weft plain stitch show great strength, good flexibility and low viscidity. Alginate is non-toxic and aids in homeostasis as a part of the wound healing process [2]. Medical dressings of alginate fiber /cotton blended yarn is contact dressing. The alginate fiber insides will form one gel layer after absorbing the effusion and the gel can separate the wound from the dressings. Cotton fibers provide enough strength to maintain the shape and they are suitable to clad the wound. Viscose is also applied in medical dressings. It is often combined with non-woven materials with higher hygroscopicity to separate the wound from short fibers of non-woven materials [3, 4].

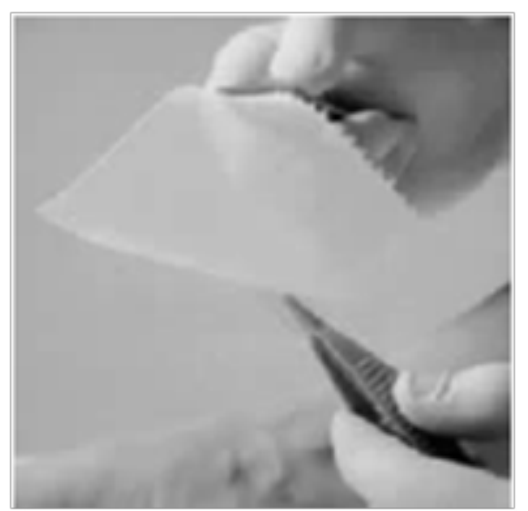

Figure 5. Viscose filament yarn knitted wound dressing fabric

\subsubsection{Weft rib stitch applied in medical dressings}

Ordinary weft rib stitch has great elasticity and extensibility when stretching along the transverse direction. And it is difficult to generate edge-roll. While jacquard stitch, which is a combination of mesh stitch and rib stitch, has properties like clear patterns, stable and thick structures, as well as lower extensibility and raveling property. And the different sizes of mesh can obtain better breathability and moisture permeability. Jacquard stitch mixes the advantages of the structures above and greatly satisfies the requirements of medical dressings.

Weft rib stitch medical dressings are bioactive dressings for the application of biological materials. It contains bioactive substances that have got the greatest attention [5]. Jutecell is a new regenerated cellulose fiber with excellent antibacterial mildew and XOPT-Dry property. It is an ecological textile fiber that is new, healthy and fashionable, and has environmental protection properties; it shows great potential in medical dressings. Chitosan [6-11] is a biological material with excellent properties of hemostatis, granulation, and epithelization. It has good biocompatibility and is suitable for wounds during various healing phases.

Medical dressings based on the rib stitch can be knitted by Jutecell/cotton blended yarn (80/20). And jacquard-rib stitch medical dressings can be created with Chitosan knitting mesh stitch and cotton knitting rib stitch. Figure 6 shows the structure of the Jacquard-rib stitch and Figure 7 shows the knitting pattern of the Jacquard-rib stitch.

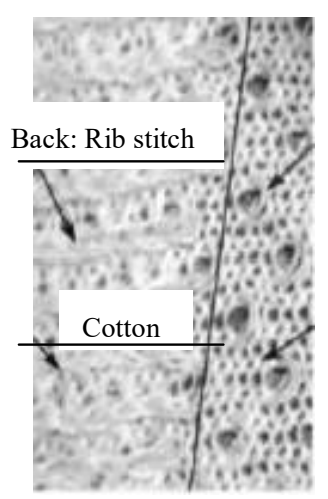

Figure 6. The structure of the Jacquard-rib stitch

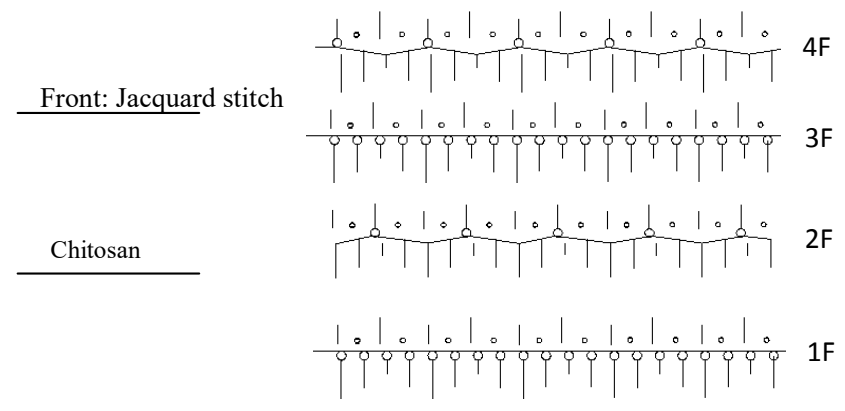

Figure 7. The knitting pattern of the Jacquard-rib stitch 


\subsubsection{Weft multiply composites applied in medical dressings}

Weft multiply composites have flexible structures with different functions. The inner layer is plain stitch with dense structures. The interlayer is fleecy stitch, which increases on thickness of the dressings. And the outer layer is tuck stitch with meshes, which increases both breathability and aesthetics of the fabric. Cotton fiber and bamboo fiber is generally used in multiply composite medical dressings. Bamboo fiber has excellent natural antibacterial properties, low density, good thermal insulation and mechanical properties, sustainability, and biodegradability. Multiply composites with bamboo fiber have better antibacterial properties than cotton fiber.

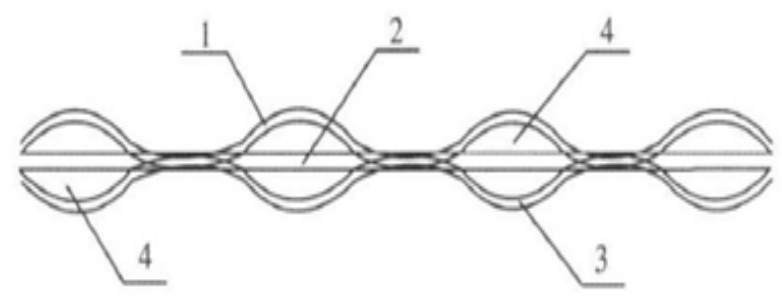

1-surface layer; 2-spacer; 3-inner layer; 4-air layer

Figure 8. The structure of the multiply composites

\subsubsection{Spacer fabrics applied in medical dressings}

The spacer fabric structure is often used as the backbone of the composite wound dressing. Spacer fabrics have threedimensional (3D) textile structure with three layers. The fabrics have good breathability with high air permeability and a good ability to control heat and moisture transfer [12-15]. It also has good ability to control the distribution of pressure. Weft knitted spacer fabrics, knitted with a 14-gauge STOLL CMS 822 computerized flat knitting machine, have better absorbency and a better thermal property. Warp-knitted spacer fabrics may be used as a substitute for the absorbent layers of wound dressings for their good linear elastic compressibility, breathability and thermal conductivity [16-18].

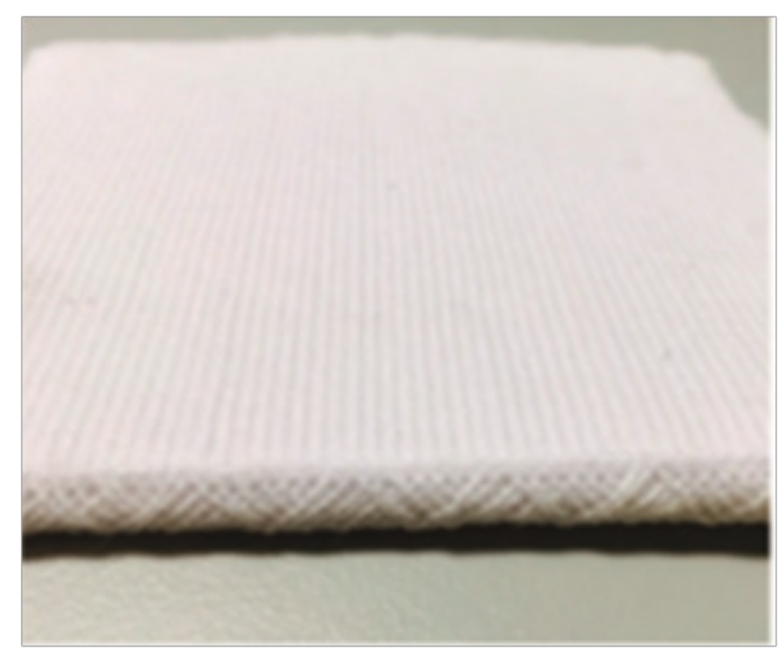

Figure 9. A typical fabricated spacer fabric

\subsubsection{Medical bandages}

Warp knitted structures, such as pillar stitch and tricot stitch, are gradually applied in medical bandages. The bandage is knitted by glass fiber on Italian COMEZ crocheting machine. Water-soluble PVA fiber also has great potential in medical bandages for its good biocompatibility and degradability. It is knitted on 18-gauges DR10 EWH double needle-bar warp knitting machine. Double needle-bar tubular structures will be given good elasticity and extensity.

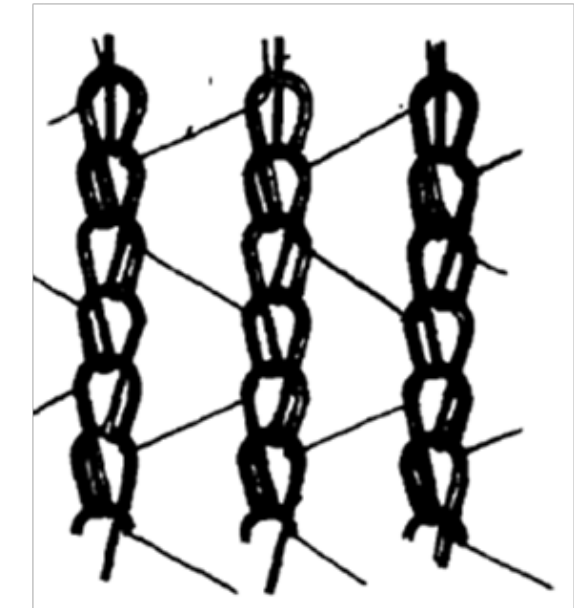

Figure 10. The structure of the bandage knitted by glass fiber

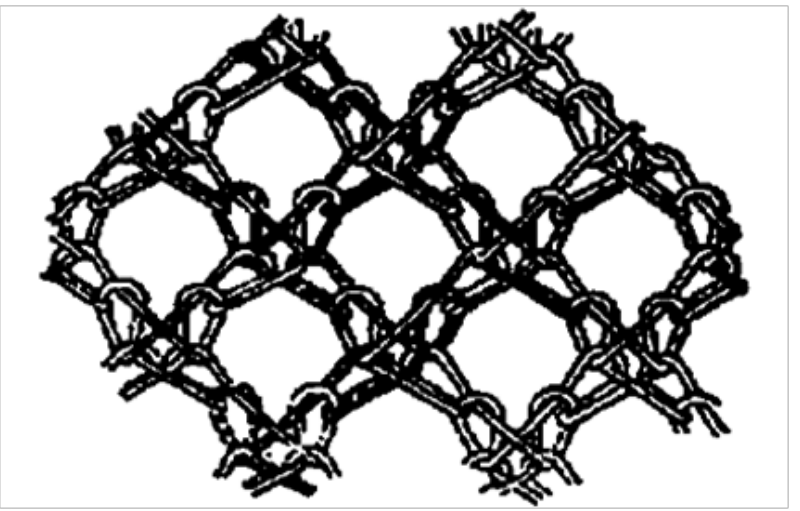

Figure 11. The structure of the bandage knitted by water-soluble PVA fiber

\subsection{Implantable textiles}

Implantable textile is a series of materials made of textile technology, which can be implanted into human bodies. It is flexible, biocompatible and easy to weave. Implantable textiles mainly include artificial blood vessels, hernia patches, artificial ligaments, etc. It can be weaved into various structures for use in the different parts of the body and different functions.

\subsubsection{Artificial blood vessel}

Warp-knitted structures are frequently-used to knit artificial blood vessels for stable structures and high compliance. And excessive expansion, edge roll and ladder rarely exit. Artificial blood vessel is knitted on double needle-bar warp knitting machine with more patterns and ground bars. Tricot stitch and atlas stitch are generally used. Figure 13 (a) shows the double 
tricot stitch and the two bars have reverse underlap movement. Figure 13 (b) shows the reverse locknit stitch. And Figure 13 (c) shows the combination of tricot stitch and atlas stitch with reverse underlap movement. Figure 12 is the actual warpknitted artificial blood vessel.

The materials applied in artificial blood vessels include polyester, polypropylene, polyethylene, polyurethane, polytetrafluoroethylene and some other synthetic fibers [19]. And silk fibroin is also used for its high strength and toughness. The specifications are changeable as it is applied in different occasions [20].

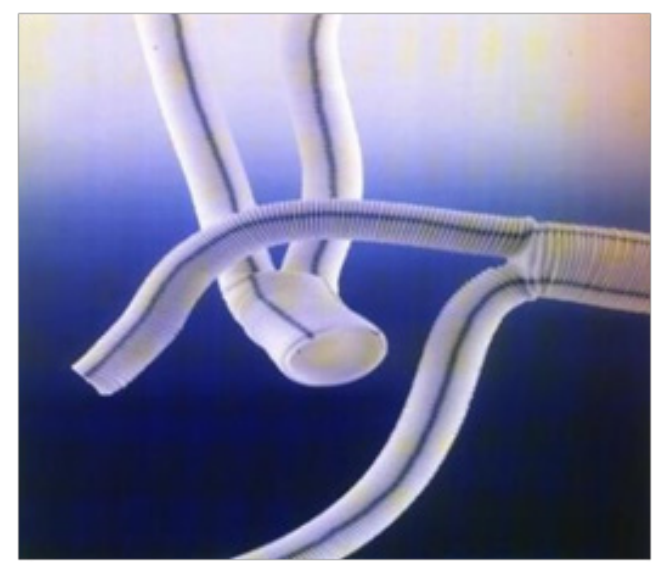

Figure 12. Actual warp-knitted artificial blood vessel

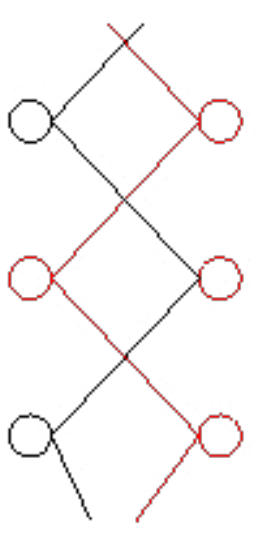

(a)

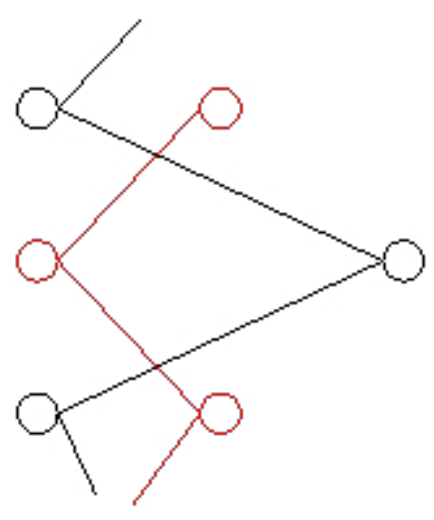

(b)

\subsubsection{Hernia patch}

Hernia patch is an important implant for hernia repair. And polymer meshes are frequently used in hernia surgery. Warpknitted pillar stitch, tricot stitch and atlas stitch are generally structures for hernia patch. Marlex® adopts tricot stitch as the basic structure and Prolene $®$ adopts atlas stitch. They all have small pores [21]. Surgipro $®$ PP hernia patch and Dexon ${ }^{\circledR}$ PGA hernia patch are created by tricot stitch and pillar stitch. The structures have higher strength and greater stability. The materials include non-absorbable polymers like polyester, polypropylene, expanded polytetrafluoroethylene, polyvinylidenefluoride and absorbable polymers polyglycolic acid [22]. Figure 14 shows two hernia patches made of polypropylene. Figure $14(\mathrm{a})$ is Marlex $®$ patch with small pores and Figure $14(\mathrm{~b})$ is Vyproß patch with large pores.

\subsubsection{Artificial ligament}

Artificial ligaments can be used to replace the damaged knee joint. Ligament advance reinforcement system (LARS) studied by Laboureau is widely used in anterior cruciate ligament reconstruction [23-25]. The material is medical polyester, and the structure is warp-knitted. As shown in Figure 15, the longitudinal fibers are bound together with a transverse knitted structure. And the longitudinal fibers are parallel and pre-twisted at $90^{\circ}$ [26]. According to the number of longitudinal fibers, the diameter of the ligament has different sizes and they

Figure 13. Some structures applied in warp-knitted artificial blood vessel

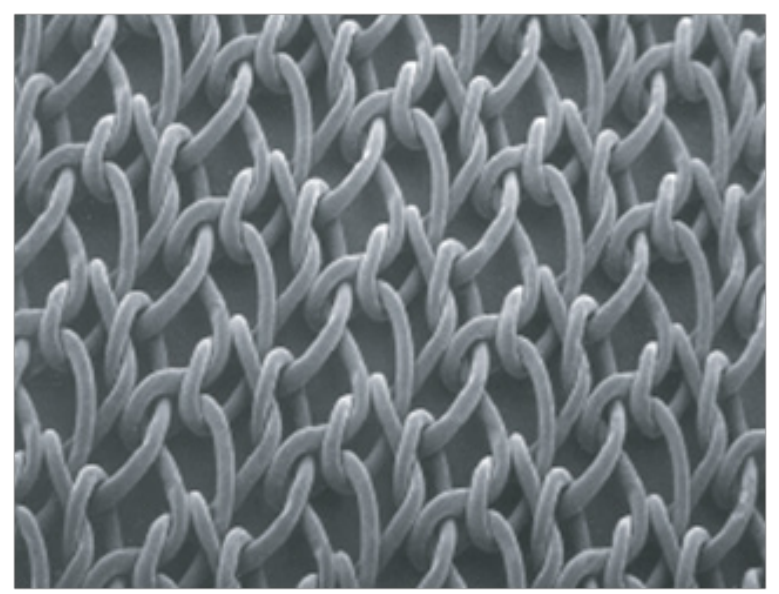

(a)

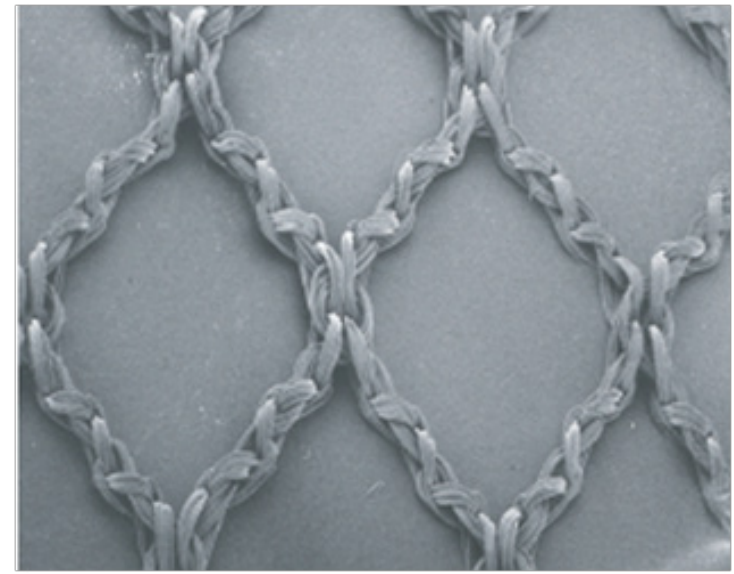

(b)

Figure 14. The actual structures of hernia patch. (a) Marlex® patch; (b) Vypro® patch

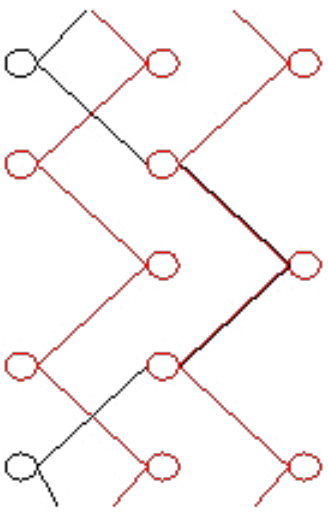

(c) 
are used for different situations [27]. LARS ligament has lower extensibility, higher tensile strength, great fatigue strength and satisfactory clinical results [28].

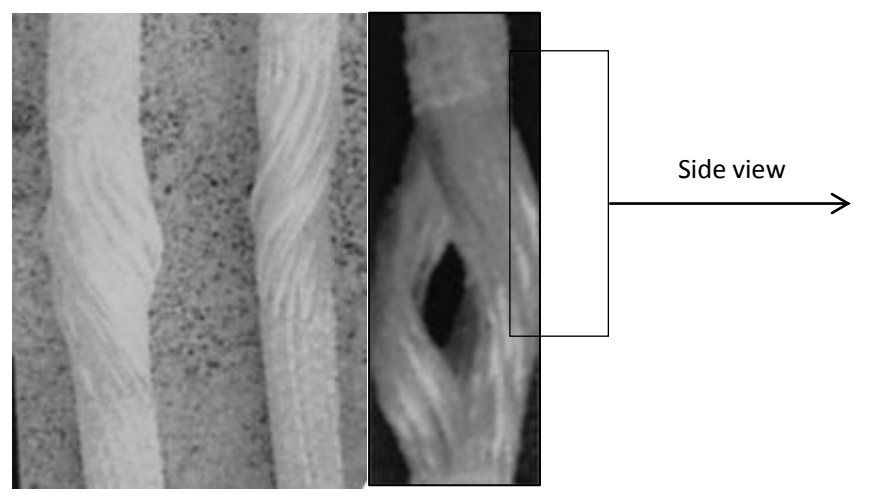

Figure 15. Ligament advance reinforcement system

\subsubsection{Cardiac Support Device}

Cardiac Support Device (CSD) is a warp-knitted elastic mesh bag, which is cut and sewn on the basis of the shape of the heart $[29,30]$. Atlas stitch is the basic structure and the material is multi-filamentous yarn to provide high-strength and fatigueresistant characteristics, while maintaining flexibility [31]. The fabric with warp-knitted atlas stitch is glossy and it is easy to slide on the surface of heart to provide acute wall support.

\subsubsection{Urethral suspension sling}

The sling used in suspension is warp-knitted mesh fabric $[32,33]$. Figure 17 (a) shows the fabric with small meshes and the structure is tricot stitch. Figure 17 (b) shows the fabric with large meshes and the structure is atlas stitch. The warp-knitted structures give the fabric certain tension strength, elasticity, durability and different sizes of meshes. Studies show that the size of the aperture will influence the clinical results. The size of the aperture increases and the softness of slings will increase. The chance of infection will decrease. The role materials include polypropylenemono filament (e.g. TVT $^{\circledR}$ ) and polypropylene multifilament monofilament (e.g., IVS ${ }^{\circledR}$ ). Both TVT $^{\circledR}$ and IVS ${ }^{\circledR}$ have bigger meshes.

\subsubsection{Knitted medical expandable metallic stent}

The use of metallic expandable tracheal stents is to provide a palliative relief for severe airway obstruction. The Ultraflex ${ }^{\mathrm{TM}}$ stent (Micro-invasive, Boston Scientific, and Watertown, MA, USA) is a kind of mesh knitted by a single strand of nitinol wire $[34,35]$. Nitinol is an alloy of $55 \%$ nickel and $45 \%$ titanium, and it has thermal memory. The stent is compressed along a supple guide by a coiled thread. If the thread is pulled, the stent will self-expand to its final diameter (Figure 15a). The Ultraflex ${ }^{\mathrm{TM}}$ stent may be in an uncovered form or covered by a sheath of silastic. The covered one is often used to prevent growth of tumor tissue (Figure 15b). And according to the difference of genders, the stent diameters for patients differ [36].

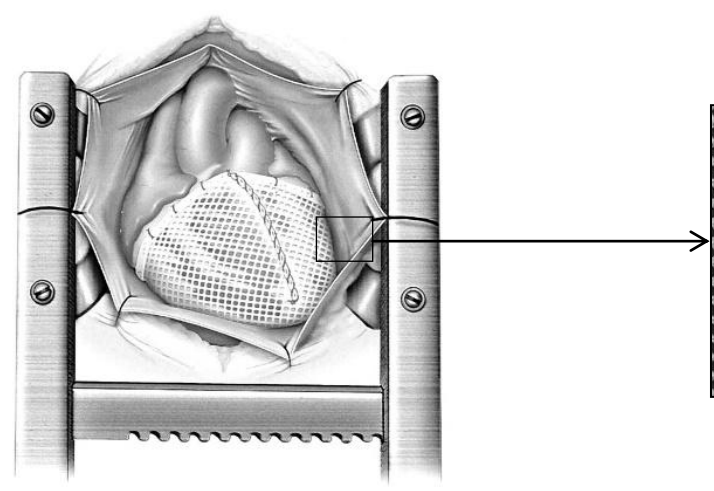

Figure 16. Positioning and securing the $\mathrm{CSD}[31]$

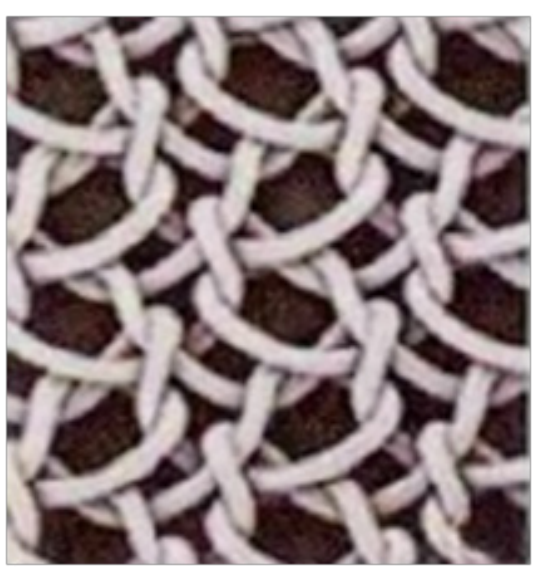

(a)

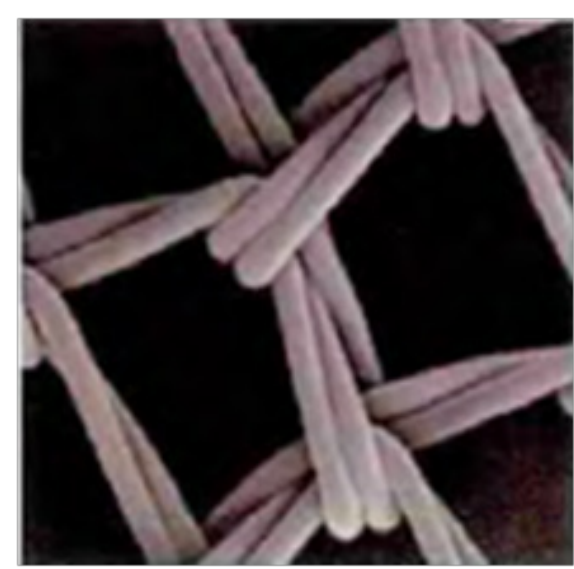

(b)

Figure 17. Urethral suspension sling. (a) Tricot stitch; (b) Atlas stitch 


\subsubsection{Artificial tracheal stent}

Artificial tracheal stent is always biodegradable and can be absorbed by human body. Artificial tracheal stent can temporarily replace the removed tracheal and maintain the airway travel-ability for easy regeneration of the tracheal.

Plain plating stitch can be used to knit artificial tracheal stent. The plating yarn is polyglycolide lactide (PLGA) and the ground is polypropylene (PP). The fabric is knitted on small diameter weft-knitting machine and it is a tubular fabric. And before being implanted in human body, the tracheal stent needs to be coated and sterilized.

\subsubsection{Periodontal guided tissue regeneration}

Periodontal patch is a kind of microporous membrane for the treatment of periodontal disease and guides the regeneration of periodontal tissue. The basic structure is plain stitch knitted on a single circular knitting machine. Biodegradable fibers, PLGA and PGA, are chosen as the materials. And according to the requirements, different shapes of the patch can be sewn.

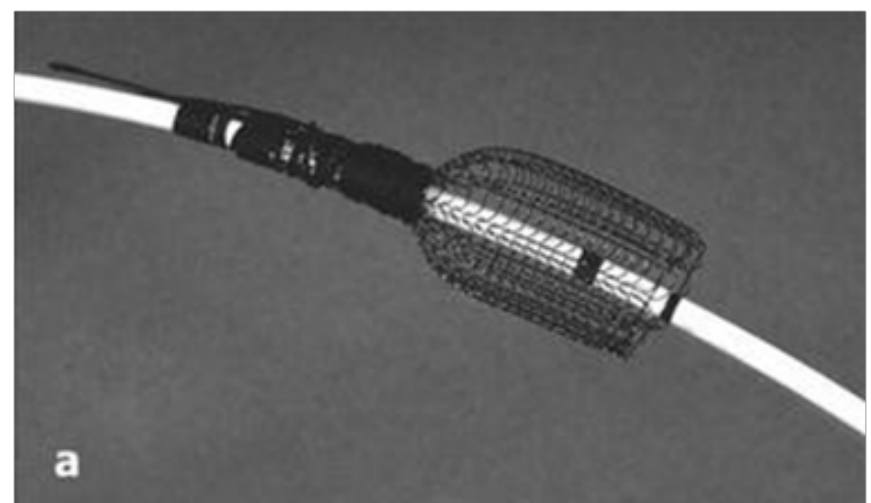

\subsubsection{Tendon scaffold}

Compared with braided fabrics, knitted scaffolds can be proven to favor deposition of collagenous connective tissue matrix and is crucial for tendon/ligament reconstruction [37]. Weft plain stitch is applied to a large extent in tendon scaffold. PLGA and PGA is the usual material for tendon scaffold. PLGA is used to knit plain stitch as the external sheath and PGA fibers keep straight as the core. And warp-knitting technology can be applied in the field of tendon scaffold. Figure 21 is the coresheath structure of the tissue engineering tendon scaffold.

\subsubsection{Artificial chest wall}

Artificial chest wall is used to a large extent in chest wall reconstruction. The two warp-knitted fabrics - tricot stitch based on rib and tricot stitch based on interlock - are chosen for their good properties to resist ladder. The materials include poly-p-dioxanone (PDS). Figure 22 and Figure 23 show the loop structure diagram and graphic record of guide bar lay-in thread movement of the tricot stitch based on rib and interlock.

Figure 18. Urethral suspension sling.[36] (a) Self-expand to the final diameter; (b) The covered one to prevent growth of tumor tissue

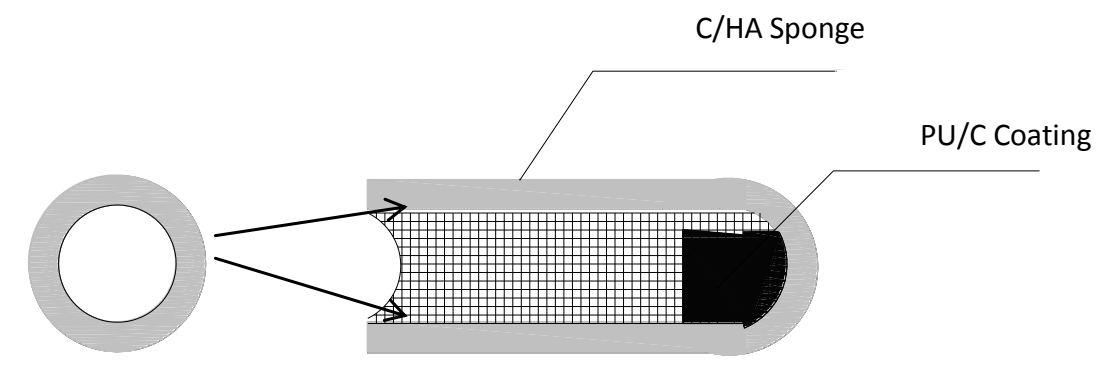

Figure 19. The structure of PP/PGLA artificial tracheal stent

(a)

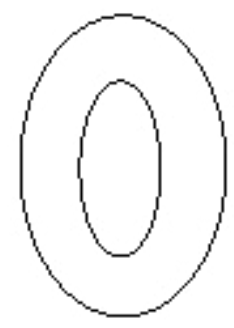

(b)

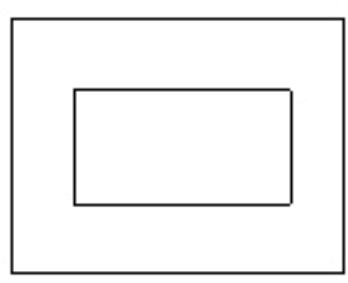

(c)

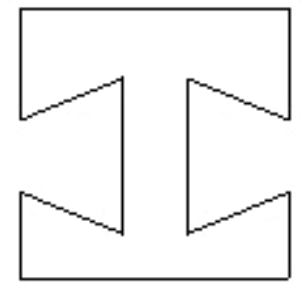

(d)

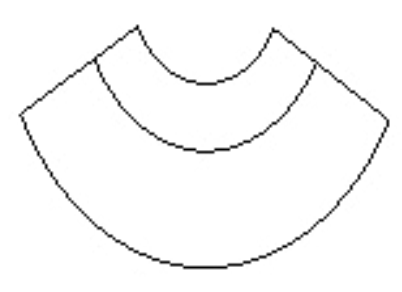

(e)

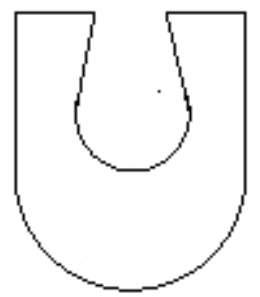

Figure 20. Some types of periodontal patches. (a) Elliptic type; ( b) Square type; ( c) adjacent surface type; ( d) Single tooth type; (e) Encircling type 


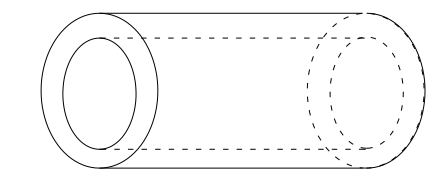

Scaffold reinforcement PGA fiber
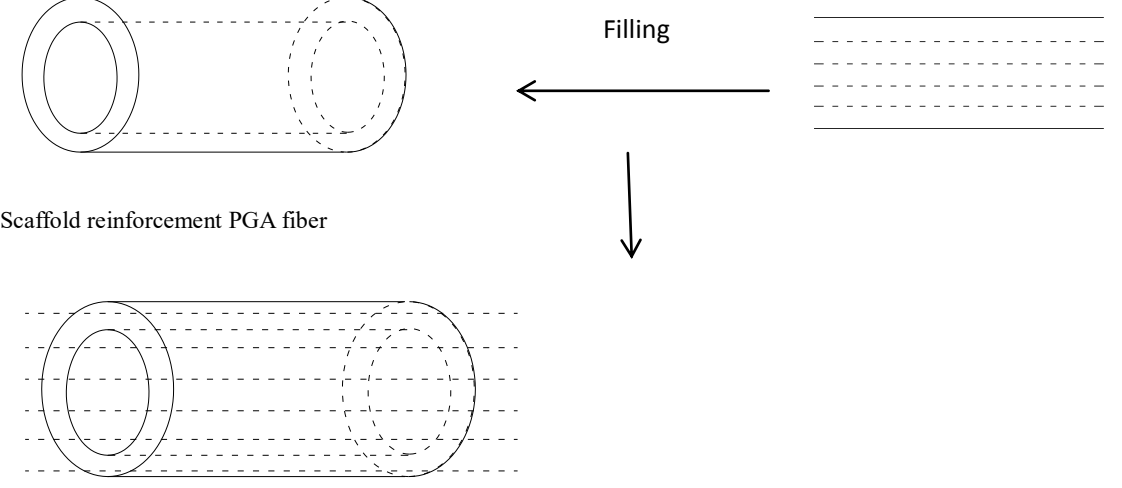

Integral reinforcement

Figure 21. The core-sheath structure of the tissue engineering tendon scaffold
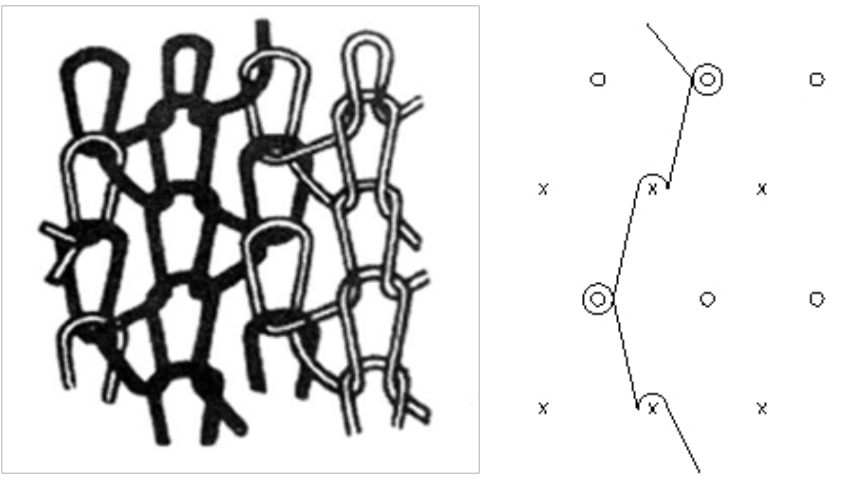

Figure 22. The loop structure diagram and graphic record of guide bar lay-in thread movement of tricot stitch based on rib
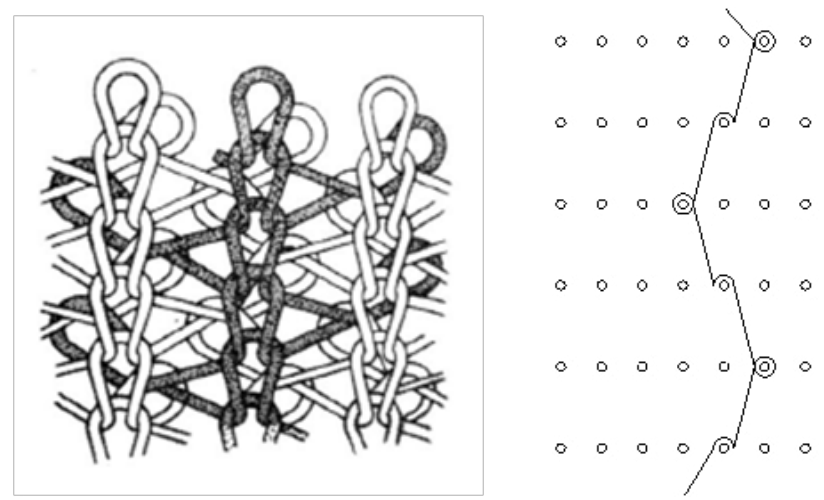

Figure 23.The loop structure diagram and graphic record of guide bar lay-in thread movement of tricot stitch based on interlock

\subsubsection{Radiotherapy stent}

Axial fabric (Figure 24) has enough strength to be applied in radiotherapy stent. Generally, weft yarn and warp yarn is inlayed in plain stitch to form axial fabric. Catheter with diamond structure is woven first on braiding machine. After coating and heat setting, the catheter is imported to the special weft-knitting machine to knit the composite structure. The materials are all biodegradable biomaterials, including collagen, polylactic acid (PLA), polyglycolic acid (PGA) and polyglycolide lactide (PGLA).

\subsubsection{Department of orthopedics splint}

Weft yarn and warp yarn can be inlayed in rib stitch to form multi-axial fabric. This kind of fabric is a multi-layered biaxial

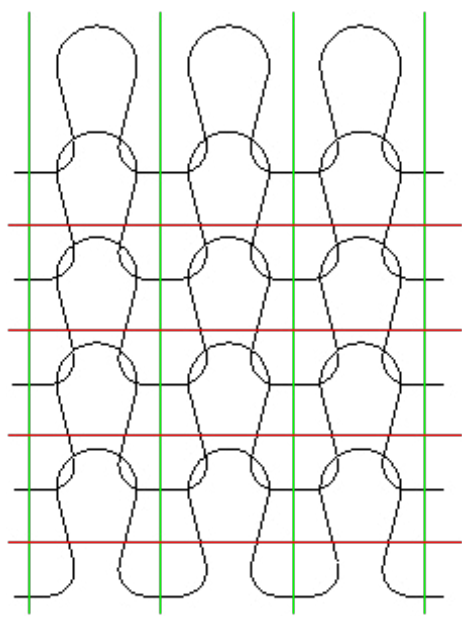

Figure 24. The axial fabric applied in radiotherapy stent 
weft knitted fabric (MBWK Fabrics). It can be used in the department of orthopedics splint, such as foot splint (Figure 25). Weft yarn and warp yarn can be glass fiber or some other high-performance fibers and the yarn knitting rib stitch can be PET or other chemical fibers. The fabric is often compounded with photosensitive resin to form the orthopedics splints in the same shapes as the injured part.

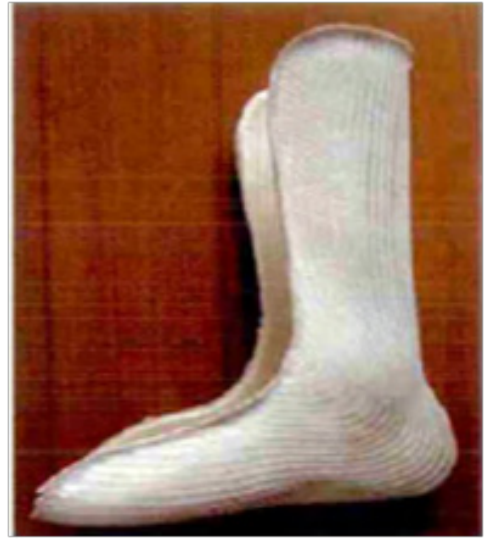

Figure 25. The Foot splint based on MBWK fabric

\subsection{Knitting structure and healthcare and hyqiene products}

Healthcare and hygiene products refer to health protection and hygiene, which involve thermostatic textiles in surgery, medical mattress, mattress covering material and some protective products.

\subsubsection{Thermostatic textiles in surgery}

Warp-knitting technology is one of the most economic and interesting technologies to create three-dimensional fabrics. A large number of warp-knitted spacer fabrics with different patterns and characteristics can be manufactured. Thermostatic textiles in surgery are such fabrics that can maintain the patient's body temperature.

A conductive yarn (a metal-coated polymer having a fabric characteristic, a carbon fiber and a metal multifilament) is added as a single yarn to the fabric during the knitting process at a specific yarn tension. These conductive elements are fed without deformation by introducing the yarn in the middle of the bars. The ground bars only vibrate during the rotation of the pattern roller, preventing the functional yarn from being woven into the fabric surface and the opposite side. The enclosed air in the spacer fabric as an insulator prevents heat from being radiated downward. As long as the side of the heating system will be close to the body, heat can be produced and absorbed by the human body without any loss. Thermostatic textiles can be widely used in medical area, especially for the prevention of hypothermia, as well as for emergency situations, such as rescue colds in the environment. Through the use of batteries for the control system for energy, heated three-dimensional warp-knitted fabric can be used to produce lightweight, flexible and reliable portable system. Figure 26 shows the theoretical position of the heat conductor in the spacer of the warp knitting structure. Figure 27 shows the new three-dimensional heating textiles.

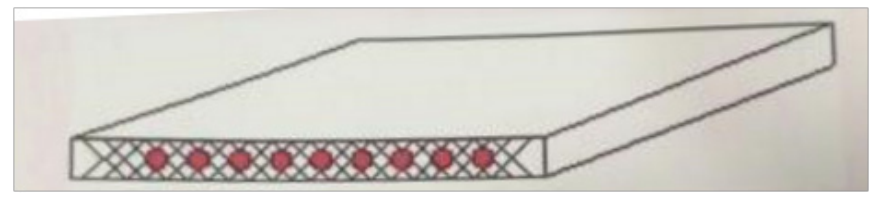

Figure 26. The theoretical position of the heat conductor in the spacer of the warp knitting structure

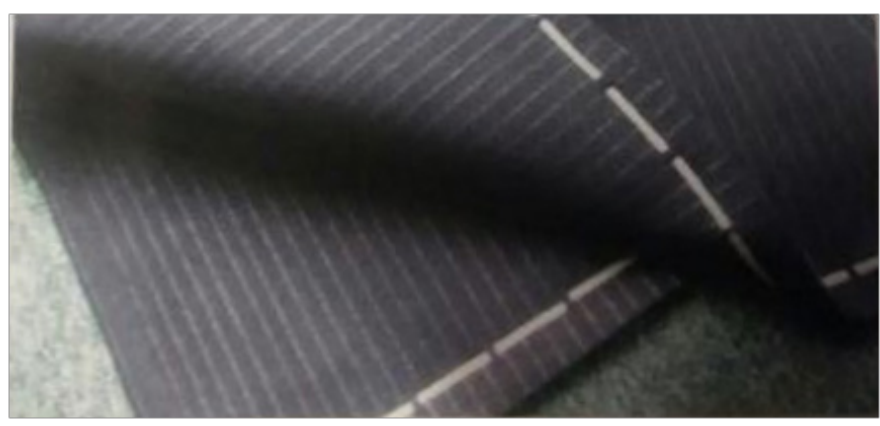

Figure 27. The new three-dimensional heating textiles

\subsubsection{Medical mattress}

Warp-knitted spacer fabric is used to a large extent as cushioning material because of its stable structure, light weight, good gas permeability, good compression and resilient behavior. Warpknitted spacer fabric is knitted on double needle bar raschel machine, and it can be used as anti-bedsore mattress, pad for surgery and mattress on wheelchair. PET with strong bending rigidity is chosen to knit the fabric. Figure 28 shows the diagram of warp-knitted mattress.

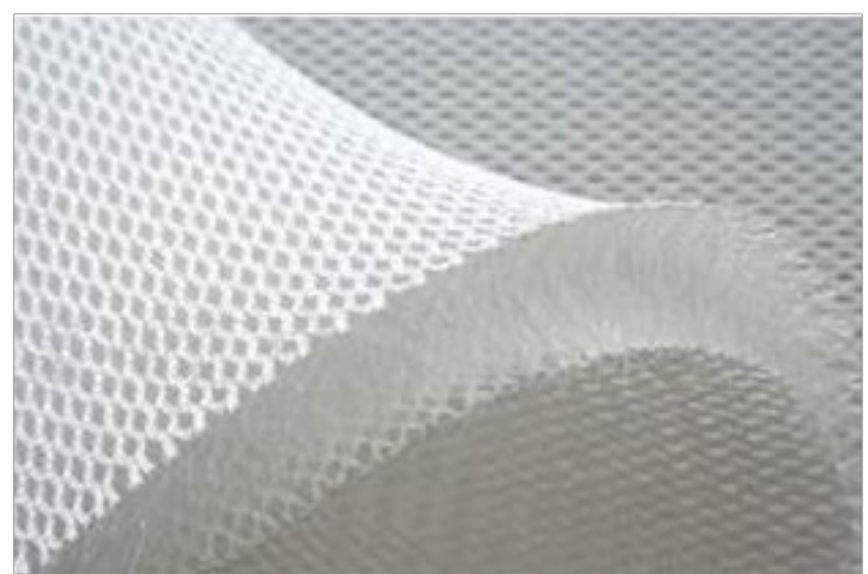

Figure 28. The diagram of warp-knitted mattress

\subsubsection{Mattress covering material}

Weft-knitted spacer fabric is knitted on circular weft knitting machine with electronic jacquard device with two surface layers and the spacer filaments connecting the two surface layers. The thickness varies from $1.5 \mathrm{~mm}$ to $5.5 \mathrm{~mm}$. Weft-knitted spacer fabric is a good mattress covering material due to its good permeability, moisture absorption, good elasticity, elastic controllability and good temperature regulation. The materials are generally PET and PA. Figure 29 shows the diagram of warp-knitted covering material. 


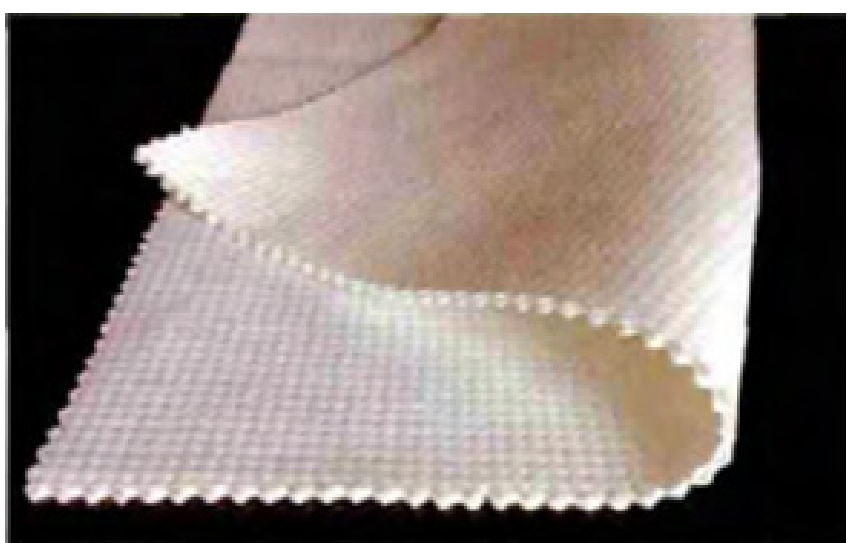

Figure 29. The diagram of warp-knitted covering material

\section{Conclusions}

Knitting technology is one of the future developments of high-tech medical textiles research and application, especially cell scaffolds in tissue engineering disciplines. The knitted medical textiles are a popular trend in technical textiles, with high added value and high technology content. A large number of structures and materials are continuously being developed to meet the requirements for medical applications. However, there are still challenging issues for those devices that are implanted in human body, since some textiles have shown tendencies to cause rejective reaction and device failure after long-term use. With increase in aging population, large number of injuries and a demand for higher quality of life, a wide range of knitted medical textiles and materials are required to fulfill some vital requirements in medical areas.

\section{Acknowledge}

The authors acknowledge the financial support from China Postdoctoral Science Foundation (2017T100325, 2016M591767), Fundamental Research Funds for the Central Universities (JUSRP51625B), Applied Foundation Research Funds of China Textile Industry Association (J201604), and Innovation Fund Project of Cooperation among Industries, Universities \& Research Institutes of Jiangsu Province (BY2015019-01).

\section{References}

[1] Czajka, R., Development of medical textile market. Fibres \& Textiles in Eastern Europe, 2005. 13(1): p. 3.

[2] Momoh, F.U., et al., Development and functional characterization of alginate dressing as potential protein delivery system for wound healing. International Journal of Biological Macromolecules, 2015. 81: p. 137-150.

[3] Turner, T., The development of wound management products. Wound: A Compendium of Clinical Research and Practice, 1989. 1(3): p. 155-171.

[4] Peršin, Z., et al., The study of plasma's modification effects in viscose used as an absorbent for wound-relevant fluids. Carbohydrate Polymers, 2013. 97(1): p. 143-151.

[5] Angspatt, A., et al., Carboxymethylchitosan, alginate and tulle gauze wound dressings: a comparative study in the treatment of partial-thickness wounds. Asian Biomedicine, 2011. 5(3): p. 413-416.
[6] Azad, A.K., et al., Chitosan membrane as a woundhealing dressing: Characterization and clinical application. Journal of Biomedical Materials Research Part B-Applied Biomaterials, 2004. 69B(2): p. 216-222.

[7] Niekraszewicz, A., Chitosan medical dressings. Fibres \& Textiles in Eastern Europe, 2005. 13(6): p. 3.

[8] Jayakumar, R., et al., Biomaterials based on chitin and chitosan in wound dressing applications. Biotechnology Advances, 2011. 29(3): p. 322-337.

[9] Muzzarelli, R.A.A., Chitins and chitosans for the repair of wounded skin, nerve, cartilage and bone. Carbohydrate Polymers, 2009. 76(2): p. 167-182.

[10] Chen, C., et al., Bubble template fabrication of chitosan/ poly(vinylalcohol) sponges for wound dressing applications. International Journal of Biological Macromolecules, 2013. 62: p. 188-193.

[11] Muzzarelli, R.A.A., Biochemical significance of exogenous chitins and chitosans in animals and patients. Carbohydrate Polymers, 1993. 20(1): p. 7-16.

[12] Liu, Y. and H. Hu, Compression property and air permeability of weft-knitted spacer fabrics. Journal of the Textile Institute, 2011. 102(4): p. 366-372.

[13] Anon, 3D warp-knitted textiles - fabrics that fit into a frame. Kettenwirk-Praxis, 2013. 04: p. 31-32.

[14] Yang, Y. and H. Hu, Spacer fabric-based exuding wound dressing - Part I: Structural design, fabrication and property evaluation of spacer fabrics. Textile Research Journal, 2016.

[15] Yang, Y. and H. Hu, Spacer fabric-based exuding wound dressing - Part II: Comparison with commercial wound dressings. Textile Research Journal, 2016.

[16] Liu, Y., et al., Impact compressive behavior of warp-knitted spacer fabrics for protective applications. Textile Research Journal, 2012. 82(8): p. 773-788.

[17] Rajan, T.P., G. Ramakrishnan, and P. Kandhavadivu, Permeability and impact properties of warp-knitted spacer fabrics for protective application. Journal of the Textile Institute, 2016. 107(9): p. 1079-1088.

[18] Tong, S.-f., et al., Exploring use of warp-knitted spacer fabric as a substitute for the absorbent layer for advanced wound dressing. Textile Research Journal, 2015. 85(12): p. 1258-1268.

[19] Nemeno-Guanzon, J.G., et al., Trends in Tissue Engineering for Blood Vessels. Journal of Biomedicine and Biotechnology, 2012. 2012: p. 1-14.

[20] Yagi, T., et al., Preparation of double-raschel knitted silk vascular grafts and evaluation of short-term function in a rat abdominal aorta. Journal of Artificial Organs, 2011. 14(2): p. 89-99.

[21] Klosterhalfen, B., K. Junge, and U. Klinge, The lightweight and large porous mesh concept for hernia repair. Expert Review of Medical Devices, 2005. 2(1): p. 103-117.

[22] Klosterhalfen, B., et al., Polymers in hernia repair - common polyester vs. polypropylene surgical meshes. Journal of Materials Science, 2000. 35(19): p. 4769-4776.

[23] Gao, K., et al., Anterior Cruciate Ligament Reconstruction With LARS Artificial Ligament: A Multicenter Study With 3- to 5-Year Follow-up. Arthroscopy: The Journal of Arthroscopic \& Related Surgery, 2010. 26(4): p. 515-523.

[24] Cheng, C., S. Ren, and P. Chen, Ligament advanced reinforcement system versus tendon autograft for anterior 
cruciate ligament reconstruction: a meta-analysis. Orthopedic Journal of China, 2016. 24(20): p. 1868-1875.

[25] Li, B., Y. Wang, and C. Qiu, Efficacy of LARS artificial ligament versus tibialis anterior allograft for posterior cruciate ligament reconstruction: a comparative study. Orthopedic Journal of China, 2016. 24(18): p. 1650-1654.

[26] Parchi, P.D., et al., Anterior cruciate ligament reconstruction with $L A R S^{\mathrm{TM}}$ artificial ligament results at a mean follow-up of eight years. International Orthopaedics, 2013. 37(8): $p$. 1567-1574.

[27] Dericks Jr, G., Ligament advanced reinforcement system anterior cruciate ligament reconstruction. Operative Techniques in Sports Medicine, 1995. 3(3): p. 187-205.

[28] Hamido, F., et al., The use of the LARS artificial ligament to augment a short or undersized ACL hamstrings tendon graft. The Knee, 2011. 18(6): p. 373-378.

[29] Starling, R.C., et al., Sustained Benefits of the CorCap Cardiac Support Device on Left Ventricular Remodeling: Three Year Follow-up Results From the Acorn Clinical Trial. The Annals of Thoracic Surgery, 2007. 84(4): $p$. 1236-1242.

[30] Konertz, W.F., et al., Passive containment and reverse remodeling by a novel textile cardiac support device. Circulation, 2001. 104(12): p. I270-1275.

[31] Oz, M.C., et al., Global surgical experience with the Acorn cardiac support device. The Journal of Thoracic and Cardiovascular Surgery, 2003. 126(4): p. 983-991.
[32] Zullo, M.A., et al., One-Year Follow-up of Tension-free Vaginal Tape (TVT) and Trans-obturator Suburethral Tape from Inside to Outside (TVT-O) for Surgical Treatment of Female Stress Urinary Incontinence: A Prospective Randomised Trial. European Urology, 2007. 51(5): $p$. 1376-1384.

[33] Gomelsky, A. and R.R. Dmochowski, Biocompatibility Assessment of Synthetic Sling Materials for Female Stress Urinary Incontinence. The Journal of Urology, 2007. 178(4): p. 1171-1181.

[34] Madden, B.P., S. Datta, and N. Charokopos, Experience with ultraflex expandable metallic stents in the management of endobronchial pathology. The Annals of Thoracic Surgery, 2002. 73(3): p. 938-944.

[35] Gonfiotti, A., et al., The first tissue-engineered airway transplantation: 5-year follow-up results. The Lancet. 383(9913): p. 238-244.

[36] Gaafar, A.H., A.Y. Shaaban, and M.S. Elhadidi, The use of metallic expandable tracheal stents in the management of inoperable malignant tracheal obstruction. European Archives of Oto-Rhino-Laryngology, 2012. 269(1): p. 247253.

[37] Sahoo, S., et al., Characterization of a novel polymeric scaffold for potential application in tendon/ligament tissue engineering. Tissue Engineering, 2006. 12(1): p. 91-99. 